\title{
Phylogenetic Modeling of Heterogeneous Gene-Expression Microarray Data from Cancerous Specimens
}

\author{
Mones S. Abu-Asab, ${ }^{1, \star}$ Mohamed Chaouchi, ${ }^{2, \star}$ and Hakima Amri²
}

\begin{abstract}
The qualitative dimension of gene expression data and its heterogeneous nature in cancerous specimens can be accounted for by phylogenetic modeling that incorporates the directionality of altered gene expressions, complex patterns of expressions among a group of specimens, and data-based rather than specimen-based gene linkage. Our phylogenetic modeling approach is a double algorithmic technique that includes polarity assessment that brings out the qualitative value of the data, followed by maximum parsimony analysis that is most suitable for the data heterogeneity of cancer gene expression. We demonstrate that polarity assessment of expression values into derived and ancestral states, via outgroup comparison, reduces experimental noise; reveals dichotomously expressed asynchronous genes; and allows data pooling as well as comparability of intra- and interplatforms. Parsimony phylogenetic analysis of the polarized values produces a multidimensional classification of specimens into clades that reveal shared derived gene expressions (the synapomorphies); provides better assessment of ontogenic pathways and phyletic relatedness of specimens; efficiently utilizes dichotomously expressed genes; produces highly predictive class recognition; illustrates gene linkage and multiple developmental pathways; provides higher concordance between gene lists; and projects the direction of change among specimens. Further implication of this phylogenetic approach is that it may transform microarray into diagnostic, prognostic, and predictive tool.
\end{abstract}

\section{Introduction}

G ENE MICROARRAY HAS BEEN EMPLOYED in studying comparative gene expression in cancer, genetic disorders, infections, drug response and interactions, as well as other biological processes (Quackenbush, 2006), and its data used to generate cancer taxonomy (Bittner et al., 2000; Golub, et al., 1999; Lossos and Morgensztern, 2006), diagnosis, prognosis (Beer, et al., 2002), subtyping/class discovery (Alizadeh, et al., 2000; Beer et al., 2002), and biomarker detection (Lossos and Morgensztern, 2006). However, after more than a decade since its introduction and subsequent wide usage, microarray gene expression is still facing a number of problems that are limiting its usefulness and potential (Harrison et al., 2007; Millenaar et al., 2006; Wang, et al., 2005). There are the problems of reproducibility of measurements between runs, instruments, or laboratories; the inability to perform intra- and interplatform comparability, pooling, and insufficient concordance of gene lists. Furthermore, there is the lack of an optimal bioinformatic tool to model the het- erogeneity of gene expression of cancerous specimens, and due to the multiphasic nature of cancer, statistically significant gene expressions are not necessarily biologically meaningful during all phases of cancer. Current analytical paradigms such as phenetic clustering and maximum likelihood (including Bayesian) have not resolved these issues (AbuAsab et al., 2008), and there is a total lack of an analytical paradigm that can transform microarray data into a multidimensional bioinformatic tool useful for a clinical setting.

Cancer incipience, progression, and maintenance are all evolutionary processes at the cellular and tissue levels; they mirror similar evolutionary processes at the population levels in that they all involve genetic modifications within an individual, selective pressure, and clonal propagation. Tumors derived from the same primary tumor become diverse and contain heterogeneous patterns of gene expression after a brief time of divergence. Data heterogeneity points out the existence of several phenomena: high genomic diversity in diseased specimens, high mutation rate, and possibly multiple pathways of disease development. To efficiently and

\footnotetext{
${ }^{1}$ Laboratory of Pathology, National Cancer Institute, National Institutes of Health, Bethesda, Maryland.

${ }^{2}$ Department of Physiology and Biophysics, School of Medicine, Georgetown University, Washington, DC.

*These authors contributed equally to this work.
} 
accurately model these phenomena, biologically compatible methods of analysis should be used.

In an attempt to resolve some of the above listed problems through biologically compatible methodology and broaden the bioinformatic potential of the microarray technology, we introduce a parsimony phylogenetic approach for microarray data analysis that is based on outgroup comparison (a.k.a. polarity assessment) and maximum parsimony. This approach is a double-algorithmic procedure where the data values are first polarized into derived or ancestral depending on whether they fall within the range of the outgroup, which is usually composed of normal healthy specimens, then the polarized data is processed with a maximum parsimony algorithm. Maximum parsimony produces a phylogenetic classification of the specimens that recognizes monophyletic classes (clades) that are delimited by shared derived gene expressions (the synapomorphies); it achieves that by finding the phylogenetic tree with the minimum steps to construct.

Biologically meaningful modeling and interpretation of the data, and better correlation with clinical characteristics, diagnosis, and outcomes are highly desired criteria in an analytical tool (Allison et al., 2006; Beer, et al., 2002; Bittner, et al., 2000; Golub, et al., 1999). Clustering specimens into unidimensional classification of discernable entities on the basis of overall quantitative gene expression similarities has some serious drawbacks (Allison et al., 2006; Lyons-Weiler et al., 2004), and appears to be incongruent with the nature of disease development (Abu-Asab, et al., 2006; 2008). In this report, we are demonstrating that the use of parsimony phylogenetic analysis of microarray data resolves the issues of gene-ranking discrepancies, improves interplatform concordance, makes possible intra- and interplatform comparability, eliminates biases in the gene linkage criteria, and casts gene expression profiles into a biologically relevant and predictive model of class discovery.

A superior classification is one that summarizes maximum knowledge about its specimens, reflects their true ontogenic relationships to one another, and offers predictivity (Farris, 1979; Golub, et al., 1999). The latter would especially be significant when the classification is applied in a clinical setting for diagnosis, prognosis, or posttreatment evaluation. We are utilizing parsimony phylogenetics because of its inherent ability to produce a robust classification of relationships-class discovery; and its forecasting power to reveal the characters of a specimen when its place in the classification is established-class prediction (Albert, 2005b). Parsimony models the heterogeneity of cancerous microarray data without any a priori assumptions (Goloboff and Pol, 2005; Siddall, 1998; Stefankovic and Vigoda, 2007). Additionally, a phylogenetic approach elucidates the direction of change among specimens that leads to their molecular and cellular diversity: the presence of one or more developmental pathway (AbuAsab et al., 2008), and novel expressions that are involved in the progression and maintenance of the disease.

A strict parsimony phylogenetic analysis uses only shared derived values, synapomorphies, to delimit a natural group of specimens within a clade (Wiley and Siegel-Causey, 1991). Shared derived values of a gene among several specimens constitute a synapomorphy; therefore, only a synapomorphy is indicative of their relatedness. Because synapomorphies define clades at various grouping levels, a parsimonious phylogenetic classification reflects hierarchical shared developmental pathways among a group of specimens and may reveal the presence of subclasses with each having its own uniquely derived gene expression synapomorphies. In biological and clinical senses, class discovery and prediction should be based on shared derived gene expressions (i.e., synapomorphies). For example, a cancer class (a clade in phylogenetic terminology) is delimited by one or more synapomorphies, and a cancerous specimen will be placed in a class only if it shares the same synapomorphies with the members of the clade.

In this study, we are describing a double-algorithmic analytical method of microarray gene-expression data based on polarity assessment algorithm, UNIPAL (Abu-Asab, et al., 2006) where the polarized values can be used by a parsimony algorithm, MIX (Felsenstein, 1989) to produce a phylogenetic classification of specimens. This approach brings in a systematic solution to class discovery through phylogenetic classification whereby every class is delimited by shared derived gene expressions-i.e., synapomorphies-delimited clades. Because such a classification reflects the shared aberrations of gene expressions of the specimens, we expect it to have a biological and clinical relevance, and to advance targeted treatments of disease.

\section{Materials and Methods}

\section{Gene expression datasets}

In order to demonstrate the applicability of parsimony phylogenetics to microarray gene expression data, and test the results of interplatform concordance and comparability, we downloaded three publicly available datasets of gene expression comparative studies, GDS484 (Hoffman et al., 2004), GDS533 (Quade et al., 2004), and GDS1210 (Hippo et al., 2002), from NCBI's Gene Expression Omnibus (http://www. ncbi.nlm.nih.gov/geo/). The GDS484 was conducted on GPL96 (Affymetrix GeneChip Human Genome U133 Array Set HG-U133A), and the other two studies on GPL80 (Affymetrix GeneChip Human Full Length Array HuGeneFL). The GDS484 was comprised of normal myometrium $(n=5)$ and uterine leiomyomas $(n=5)$ obtained from fibroid afflicted patients. The GDS533 study encompassed normal myometrium $(n=4)$, benign uterine leiomyoma $(n=7)$, as well as malignant uterine $(n=9)$ and extrauterine $(n=4)$ leiomyosarcoma specimens. The GDS1210 study included expression profiling of 22 primary advanced gastric cancer tissues and 8 normal specimens.

\section{Polarity assessment and parsimony analysis}

Polarity assessment through outgroup comparison does not use comparison of means and folds but rather it converts the continuous values into discontinuous ones through the assessment of each gene's values against that of the normals' range and produces a matrix of polarized values (0s and 1s). Our polarity assessment program, UNIPAL, compares independently each gene's value of experimental specimens against its corresponding range within the outgroup, and scores each as either derived (1) or ancestral (0), so the matrix of gene expression values is transformed into a matrix of polarized scores (0s and 1s). 
We used all the expression data points of all specimens in the analysis without any a priori selection of only a specific cluster of data. For polarity assessment (apomorphic [or derived] versus plesiomorphic [or ancestral]), data was polarized with our customized algorithm (UNIPAL) that recognized derived values of each gene when compared with the outgroups (Abu-Asab et al., 2006). Outgroups here were composed of normal healthy specimens only. Ideally, the outgroup should be large enough to encompass the maximum variation within normal healthy population. UNIPAL is freely available for noncommercial use from the authors.

The phylogenetic analysis was carried out with MIX, the maximum parsimony program of PHYLIP ver. 3.57c (Felsenstein, 1989), to produce separate parsimony phylogenetic analyses for each dataset, and the inclusive matrix of the two sets (GDS533 and GDS1210), which included all their specimens. MIX was run in randomized and nonrandomized inputs, and no significant differences were observed between the two options.

Phylogenetic trees were drawn using TreeView (Page, 1996).

\section{Interplatform concordance and comparability}

To test interplatform concordance when analyzed parsimoniously, we compared the synapomorphies of the two uterine leiomyoma datasets, GDS484 and GDS533, and recorded the percentage of concordance.

To test interplatform comparability (i.e., whether datasets can be pooled together for a parsimony analysis), we combined the polarized matrices of the two identical platform datasets, GDS533 and GDS1210, processed the combined matrix by MIX, and compared the result to their separate cladograms.

\section{Results}

The implications of a parsimonious analysis of the gene expression data are realized at several aspects: the recognition and utilization of partially asynchronous genes and dichotomously expressed asynchronous genes; the implications of outgroup selection and its effect on significant gene listing, better interplatform concordance and comparability, as well as the practical usefulness of the multidimensional cladograms.

\section{Dichotomously expressed asynchronous (DEA) genes}

Our analysis identified a specific punctuated pattern of gene expression that seemed to occur only in a set of specimens where a gene's expression values were around the normals' distribution (over and underexpressed), but did not overlap with it (Tables $1-7$ ). This pattern has been only recognized once in the literature but was not named (LyonsWeiler et al., 2004); we termed this phenomenon dichotomous asynchronicity to reflect its two-tailed distribution and deviation from the normal expression range.

Although $t$-statistic and fold-change may dismiss these asynchronous genes from the list of differentially expressed genes, or misrepresent their significance (Lyons-Weiler et al., 2004), an outgroup polarity assessment will assess each value as derived and let the parsimony algorithm reveal its significance in relation to the rest of the genes. A parsimony phylogenetic algorithm uses the polarized values of all genes to produce the most parsimonious classification, the one with the lowest number of reversals and parallelisms (i.e., minimizes multiple origins of expression states in hypothesizing the relationships among the specimens) (Albert, 2005a; Felsenstein, 2004).

Through polarity assessment a large number of DEA genes were recognized. All these genes had their expression values above and below that of the normal specimens' range, that is, derived in relation to outgroups thus pointing out the heterogeneity that exists among specimens. DEA genes were found in all the three datasets studied here (Tables 1-7), and were included within all the analyses.

\section{Most parsimonious cladograms}

Parsimony analysis produced one most parsimonious cladogram (having the least number of steps in constructing a classification of specimens) for the uterine GDS533 dataset (Fig. 1). The topology of the tree showed one large inclusive clade that encompassed all of the leiomyomas and leiomyosarcomas delimited by 32 synapomorphies (Table 1), a terminal clade with nine sarcoma specimens, middle sarcoma clade with four specimens, five small basal leiomyoma clades in tandem arrangement, followed by four basal normal clades.

The cladogram in Figure 1 showed that the leiomyoma specimens did not form a natural group by themselves-they did not form their own clade separating them from the leiomyosarcomas, and there were no synapomorphies circumscribing them as a clade when the ingroup was composed of leiomyoma and leiomyosarcoma and the outgroup composed of the normals. However, the leiomyomas shared 146 synapomorphies distinguishing them from the normals (Table 2).

The 13 leiomyosarcoma specimens separated into a large terminal clade that was delimited by 20 synapomorphies in comparison with an outgroup composed of leiomyoma and normal specimens (Table 3 ), and 29 synapomorphies derived in relation to leiomyomas only as an outgroup (Table 4). Extrauterine sarcoma specimens did not assemble together, but rather were scattered within the sarcoma clades (denoted by * on the cladogram in Fig. 1). When the leiomyomas were removed from the comparison, there were 156 synapomorphies delimiting the sarcomas (Table 5).

The various combinations of comparisons (several outgroup and ingroup compositions) illustrate the effect of outgroup and ingroup selections on the results (Tables 1-5). These comparisons also show the similarities and differences between two diseases that arise within the same tissue, as well as the relationship between the leiomyoma and leiomyosarcoma; and the possibility of the latter arising within leiomyoma.

For the gastric dataset, GDS1210, parsimony analysis produced one most parsimonious cladogram (Fig. 2). The cladogram topology showed two terminal clades with six and five specimens, respectively, and a tandem arrangement of six small clades with the largest having three specimens. The inclusive gastric cancer clade was circumscribed by 34 synapomorphies (Table 6). In a list by list comparison, our 34 identified synapomorphies for the gastric cancer overlapped only with one common gene (CST4) from the gene list of the authors of the study (Hippo et al., 2002). 
Table 1. Synapomorphies Defining a Clade of Leiomyoma and Leiomyosarcoma Specimens in Comparison to Normal Specimens (GDS533)

\begin{tabular}{|c|c|c|}
\hline \multicolumn{3}{|c|}{ A. Overexpressed synapomorphic genes: } \\
\hline D00596 & TYMS thymidylate synthetase & $\mathrm{OE}[1,2]$ \\
\hline \multicolumn{3}{|c|}{ B. Underexpressed synapomorphic genes: } \\
\hline L19871 & ATF3 activating transcription factor 3 & $\mathrm{UE}[1,2]$ \\
\hline U62015 & CYR61 cysteine-rich, angiogenic inducer, 61 & $\mathrm{UE}[1,2]$ \\
\hline X68277 & DUSP1 dual specificity phosphatase 1 & $\mathrm{UE}[1,2]$ \\
\hline V01512 & FOS v-fos FBJ murine osteosarcoma viral oncogene homolog & UE[1], NS [2] \\
\hline L49169 & FOSB FBJ murine osteosarcoma viral oncogene homolog B & $\mathrm{NS}[1], \mathrm{UE}[2]$ \\
\hline J04111 & JUN v-jun sarcoma virus 17 oncogene homolog (avian) & $\mathrm{UE}[1,2]$ \\
\hline Y00503 & KRT19 keratin 19 & $\mathrm{UE}[1], \mathrm{NS}[2]$ \\
\hline $\mathrm{U} 24488$ & TNXB tenascin $\mathrm{XB}$ & $\mathrm{UE}[1], \mathrm{UE}, \mathrm{OE}[2]$ \\
\hline \multicolumn{3}{|c|}{ C. Dichotomously-expressed synapomorphic genes: } \\
\hline M31994 & ALDH1A1 aldehyde dehydrogenase 1 family, member A1 & $\mathrm{UE}[1], \mathrm{NS}[2]$ \\
\hline X05409 & ALDH2 aldehyde dehydrogenase 2 family (mitochondrial) & NS $[1,2]$ \\
\hline D25304 & $\begin{array}{l}\text { ARHGEF6 Rac/Cdc42 guanine nucleotide exchange factor } \\
\text { (GEF) } 6\end{array}$ & $\mathrm{NS}[1,2]$ \\
\hline K03430 & C1QB complement component 1 , q subcomponent, B chain & $\mathrm{NS}[1], \mathrm{OE}[2]$ \\
\hline U60521 & CASP9 caspase 9 , apoptosis-related cysteine peptidase & $\mathrm{NS}[1,2]$ \\
\hline M73720 & CPA3 carboxypeptidase A3 (mast cell) & $\mathrm{NS}[1,2]$ \\
\hline $\begin{array}{l}\text { HG2663- } \\
\text { HT2759_at }\end{array}$ & Cpg-Enriched DNA, Clone S19 (HG3995-HT4265) & NS[1, 2] \\
\hline M14676 & FYN oncogene related to SRC, FGR, YES & $\mathrm{NS}[1,2]$ \\
\hline M34677 & F8A1 coagulation factor VIII-associated (intronic transcript) 1 & $\mathrm{OE}, \mathrm{UE}[2]$ \\
\hline U60061 & FEZ2 fasciculation and elongation protein zeta 2 (zygin II) & $\mathrm{NS}[1,2]$ \\
\hline U86529 & $\begin{array}{l}\text { GSTZ1 glutathione transferase zeta } 1 \text { (maleylacetoacetate } \\
\text { isomerase) }\end{array}$ & NS $[1,2]$ \\
\hline $\begin{array}{l}\text { HG358- } \\
\text { HT358 at }\end{array}$ & Homeotic Protein 7, Notch Group (HG358-HT358) & NS[2] \\
\hline AB002365 & $\begin{array}{l}\text { KIAA0367 BCH motif-containing molecule at the carboxyl } \\
\text { terminal region } 1\end{array}$ & NS[1], OE,UE[2] \\
\hline U37283 & MFAP5 microfibrillar associated protein 5 & $\mathrm{NS}[1,2]$ \\
\hline $\begin{array}{l}\text { HG406- } \\
\text { HT406_at }\end{array}$ & $\begin{array}{l}\text { MFI2 antigen p97 (melanoma associated) identified by } \\
\text { monoclonal antibodies } 133.2 \text { and } 96.5\end{array}$ & NS $[1,2]$ \\
\hline M55593 & $\begin{array}{l}\text { MMP2 matrix metallopeptidase } 2 \text { (gelatinase } \mathrm{A}, 72 \mathrm{kDa} \\
\text { gelatinase, } 72 \mathrm{kDa} \text { type IV collagenase) }\end{array}$ & NS[1], OE,UE[2] \\
\hline M76732 & MSX1 msh homeobox homolog 1 & $\mathrm{NS}[1,2]$ \\
\hline L48513 & PON2 paraoxonase 2 & $\mathrm{NS}[1,2]$ \\
\hline U77594 & $\begin{array}{l}\text { RARRES2 retinoic acid receptor responder (tazarotene } \\
\text { induced) } 2\end{array}$ & NS[1, 2] \\
\hline M11433 & RBP1 retinol binding protein 1 & $\mathrm{NS}[1,2]$ \\
\hline L03411 & RDBP RD RNA binding protein & $\mathrm{NS}[1], \mathrm{OE}[2]$ \\
\hline Z29083 & TPBG trophoblast glycoprotein & $\mathrm{NS}[1,2]$ \\
\hline S73591 & TXNIP thioredoxin interacting protein & NS $[1,2]$ \\
\hline
\end{tabular}

Synapomorphies include: OE gene, 8 UE genes, and 23 DE genes. Last column reports the status of the synapomorphies as described by [1] Hoffman et al. (2004) and [2] Quade et al. (2004) in their significant genes' lists. DE = dichotomously-expressed; NS = not significant; OE = overexpressed; $\mathrm{UN}=$ underexpressed.

\section{Interplatform concordance}

Testing of interplatform concordance was carried out by comparing the two lists of synapomorphies obtained from two leiomyoma studies, GDS484 and GDS533 (comparison results are summarized in Tables 7 and 8 ). Out of the $\sim 22,000$ genes in the GDS484 dataset, our analysis produced a total of 1485 synapomorphic genes circumscribing the leiomyoma specimens. Although the leiomyomas of the GDS533 were delimited by 146 synapomorphies out of $\sim 7,000$ gene probes, a comparison between the two sets of leiomyomas' synapomorphies produced 45 shared ones between the two (Tables 7 and 8 ), a 31\% concordance in synapomorphies despite the sizable difference in the num- ber of probes between the two datasets, which is still better than the $12 \%$ concordance between the statistically produced gene lists of the two published studies (Hoffman et al., 2004; Quade et al., 2004).

Furthermore, $48 \%$ concordance resulted when comparing the 32 synapomorphies of the leiomyomas and leiomyosarcomas clade (GDS533; Table 1) with the 1485 synapomorphies of the leiomyomas of GDS484 (Table 7); the clades' synapomorphies overlapped as follows: 1/1 OE, 7/8 UE (except FOSB), and $8 / 23 \mathrm{DE}$, an $89 \%$ concordance within the OE and UE and 35\% within the DE. Additionally, there was $45 \%$ concordance between the 32 synapomorphies of the leiomyomas and leiomyosarcomas clade and the gene list of Quad et al. (2004) (Table 8). 


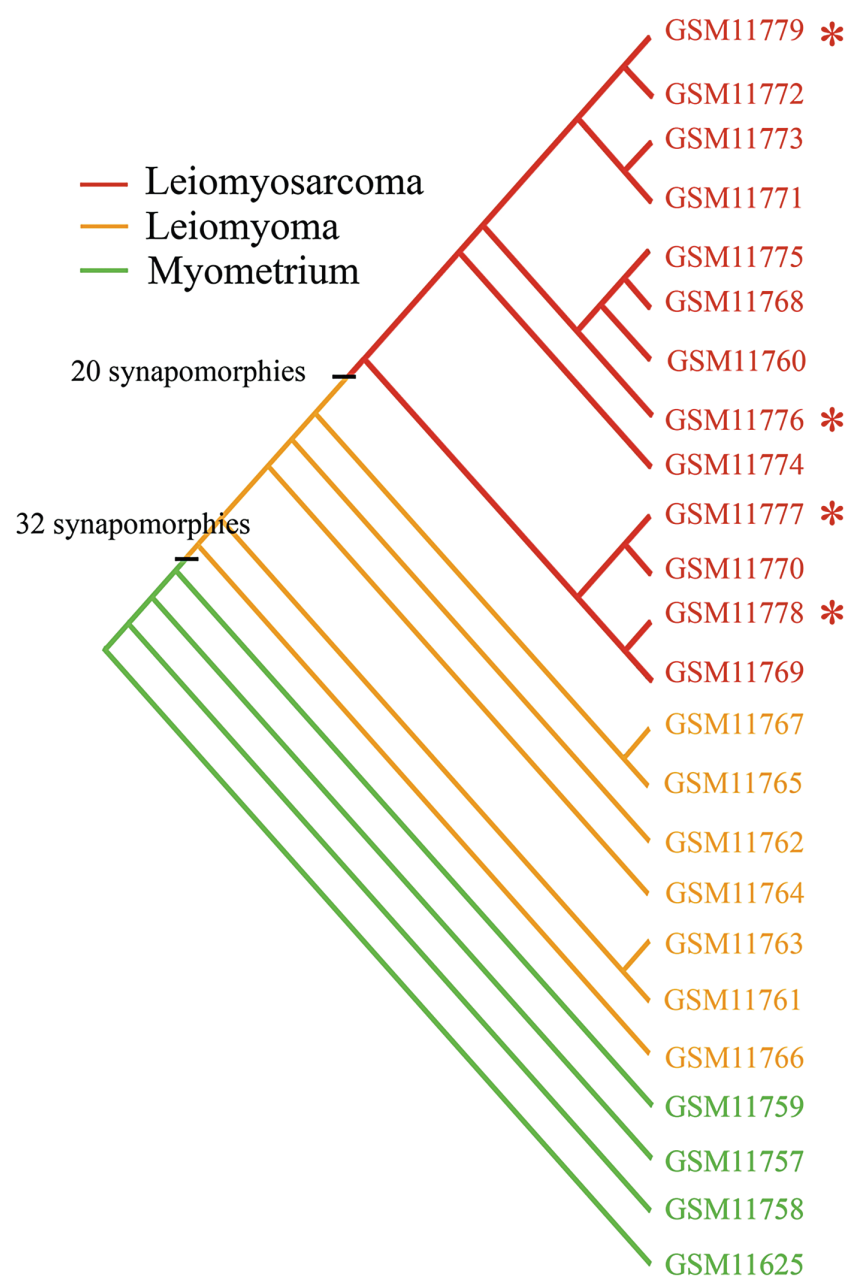

FIG. 1. A cladogram of a parsimony phylogenetic analysis of microarray gene-expression data representing normal myometrium $(n=4)$, leiomyoma $(n=7)$, leiomyosarcoma $(n=$ $9)$, and extrauterine leiomyosarcoma $(n=4)$ specimens. The leiomyomas and leiomyosarcomas form a clade defined by 32 synapomorphies (Table 1 ). The leiomyosarcoma specimens form a terminal clade that is circumscribed by 20 synapomorphies (Table 3 ). Asterisk ${ }^{*}$ ) denotes extrauterine leiomyosarcomas.

However, a lower concordance was obtained when comparing the phylogenetic synapomorphies against statistically generated gene lists. The synapomorphies of leiomyomas (GDS533; Table 2 ) showed $18 \%$ concordance $(4 / 25 \mathrm{OE}, 8 / 42$ UE) with the 78 significant genes of Hoffman et al. (2004; GDS484, gene list produced by fold change), and 16.5\% (5/25 OE, 6/42 UE) with the 146 genes of Quad et al. (2004; GDS533, gene list produced by an $F$ statistic). This was higher than the concordance between the two gene lists of the published uterine studies, $12 \%(3 / 25 \mathrm{OE}, 5 / 42 \mathrm{UE})$. The two studies had no mention of DE genes.

\section{Data pooling and interplatform comparability}

Data pooling and interplatform comparability was carried out on the combined polarized matrices of the gastric (GDS1210) and uterine (GDS533) datasets. Their inclusive parsimony analysis produced one most parsimonious clado-

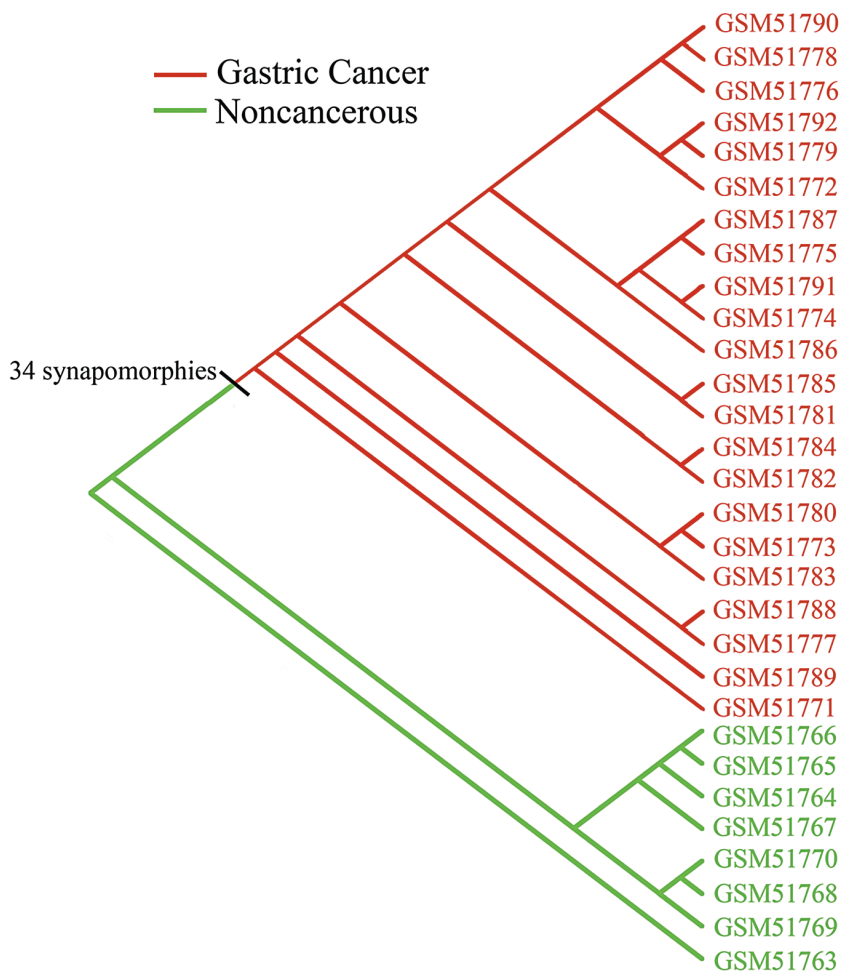

FIG. 2. A cladogram of a parsimony phylogenetic analysis of gastric cancer and noncancerous specimens. It shows a clade delineated by 34 synapomorphies (Table 6) encompassing all cancer specimens.

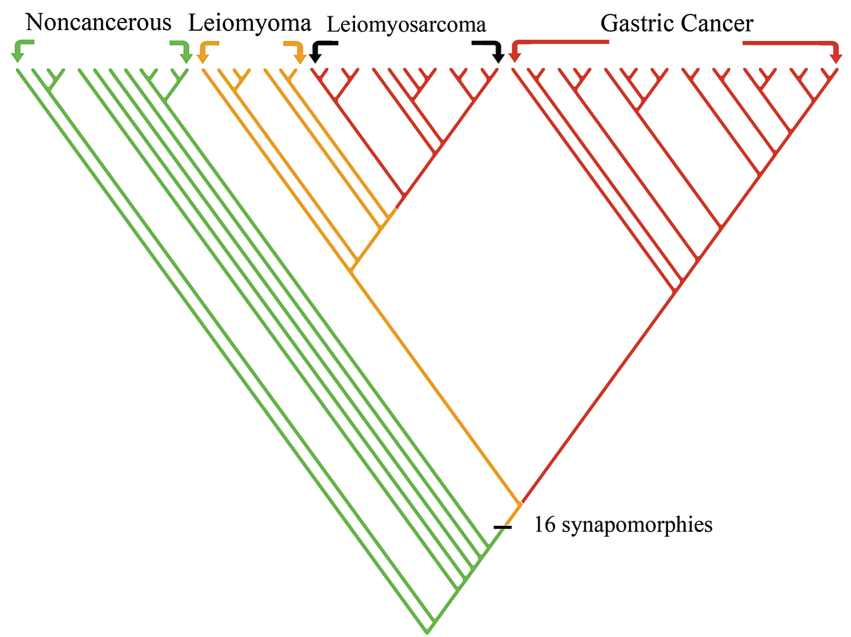

FIG. 3. A cladogram representing a comparability analysis of the gastric (GDS1210) and uterine (GDS533) datasets. The polarized matrices of the two datasets were pooled together and processed by the parsimony phylogenetic algorithm, MIX. Each of the cancers (gastric and leiomyosarcoma) forms its own clade, and the inclusive clade encompassing the two cancers and leiomyomas is delimited by a set of synapomorphies (Table 9). 
Table 2. Synapomorphies of Leiomyoma Specimens in Comparison to Normal Specimens (GDS533)

\begin{tabular}{|c|c|c|}
\hline \multicolumn{3}{|c|}{ A. Overexpressed synapomorphic genes: } \\
\hline D16469 & $\begin{array}{l}\text { ATP6AP1 ATPase, } \mathrm{H}+\text { transporting, lysosomal accessory } \\
\text { protein } 1\end{array}$ & $\mathrm{NS}[1,2]$ \\
\hline U07139 & CACNB3 calcium channel, voltage-dependent, beta 3 subunit & $\mathrm{NS}[1], \mathrm{OE}[2]$ \\
\hline M11718 & COL5A2 collagen, type $\mathrm{V}$, alpha 2 & NS $[1,2]$ \\
\hline U18300 & DDB2 damage-specific DNA binding protein $2,48 \mathrm{kDa}$ & NS $[1,2]$ \\
\hline D38550 & E2F3 E2F transcription factor 3 & $\mathrm{NS}[1,2]$ \\
\hline M34677 & F8A1 coagulation factor VIII-associated (intronic transcript) 1 & NS $[1,2]$ \\
\hline D89289 & FUT8 fucosyltransferase 8 (alpha $(1,6)$ fucosyltransferase) & $\mathrm{NS}[1,2]$ \\
\hline D86962 & GRB10 growth factor receptor-bound protein 10 & $\mathrm{NS}[1,2]$ \\
\hline M32053 & H19, imprinted maternally expressed untranslated mRNA & $\mathrm{NS}[1,2]$ \\
\hline U07664 & HLXB9 homeobox HB9 & $\mathrm{OE}[1,2]$ \\
\hline D87452 & IHPK1 inositol hexaphosphate kinase 1 & $\mathrm{NS}[1,2]$ \\
\hline U51336 & ITPK1 inositol 1,3,4-triphosphate 5/6 kinase & $\mathrm{NS}[1,2]$ \\
\hline ABA002365 & KIAA0367 & NS[1], OE[2] \\
\hline D78611 & MEST mesoderm specific transcript homolog (mouse) & $\mathrm{OE}[1], \mathrm{NS}[2]$ \\
\hline U19718 & MFAP2 microfibrillar-associated protein 2 & NS $[1,2]$ \\
\hline M55593 & $\begin{array}{l}\text { MMP2 matrix metallopeptidase } 2 \text { (gelatinase } \mathrm{A}, 72 \mathrm{kDa} \\
\text { gelatinase, } 72 \mathrm{kDa} \text { type IV collagenase) }\end{array}$ & $\mathrm{OE}[1,2]$ \\
\hline U79247 & PCDH11X protocadherin 11 X-linked & $\mathrm{NS}[1,2]$ \\
\hline L24559 & POLA2 polymerase (DNA directed), alpha 2 (70 kDa subunit) & $\mathrm{NS}[1,2]$ \\
\hline M65066 & $\begin{array}{l}\text { PRKAR1B protein kinase, cAMP-dependent, regulatory, type I, } \\
\text { beta }\end{array}$ & NS $[1,2]$ \\
\hline D14694 & PTDSS1 phosphatidylserine synthase 1 & $\mathrm{NS}[1,2]$ \\
\hline $\mathrm{U} 24186$ & RPA4 replication protein $\mathrm{A} 4,34 \mathrm{kDa}$ & $\mathrm{NS}[1,2]$ \\
\hline U85658 & $\begin{array}{l}\text { TFAP2C transcription factor AP-2 gamma (activating } \\
\text { enhancer binding protein } 2 \text { gamma) }\end{array}$ & NS $[1,2]$ \\
\hline D82345 & TMSL8 thymosin-like 8 & $\mathrm{NS}[1,2]$ \\
\hline D85376 & TRHR thyrotropin-releasing hormone receptor & $\mathrm{NS}[1,2]$ \\
\hline D00596 & TYMS* thymidylate synthetase & $\mathrm{OE}[1,2]$ \\
\hline \multicolumn{3}{|c|}{ B. Underexpressed synapomorphic genes: } \\
\hline X0330 & ADH1B alcohol dehydrogenase IB (class I), beta polypeptide & $\mathrm{NS}[1,2]$ \\
\hline M31994 & ALDH1A1* aldehyde dehydrogenaes 1 family, member A1 & $\mathrm{UE}[1], \mathrm{NS}[2]$ \\
\hline X05409 & ALDH2* aldehyde dehydrogenase 2 family (mitochondrial) & NS[1, 2] \\
\hline L19871 & ATF3* $^{*}$ activating transcription factor 3 & $\mathrm{UE}[1,2]$ \\
\hline U60521 & CASP9 caspase 9 , apoptosis-related cysteine peptidase & $\mathrm{NS}[1,2]$ \\
\hline D49372 & CCL11 chemokine ( $\mathrm{C}-\mathrm{C}$ motif) ligand 11 & $\mathrm{NS}[1,2]$ \\
\hline X05323 & CD200 molecule & $\mathrm{NS}[1,2]$ \\
\hline M83667 & CEBPD CCAAT/enhancer binding protein $(\mathrm{C} / \mathrm{EBP})$, delta & $\mathrm{NS}[1,2]$ \\
\hline U90716 & CXADR coxsackie virus and adenovirus receptor & $\mathrm{NS}[1,2]$ \\
\hline M21186 & CYBA cytochrome b-245, alpha polypeptide & $\mathrm{NS}[1,2]$ \\
\hline U62015 & CYR61* cysteine-rich, angiogenic inducer, 61 & $\mathrm{UE}[1,2]$ \\
\hline Z22865 & DPT dermatopontin & $\mathrm{NS}[1,2]$ \\
\hline X56807 & DSC2 desmocollin 2 & $\mathrm{NS}[1,2]$ \\
\hline X68277 & DUSP1* dual specificity phosphatase 1 & $\mathrm{UE}[1,2]$ \\
\hline V01512 & $\begin{array}{l}\text { FOS* v-fos FBJ murine osteosarcoma viral oncogene } \\
\text { homolog }\end{array}$ & UE[1], NS[2] \\
\hline L49169 & FOSB FBJ murine osteosarcoma viral oncogene homolog B & NS[1], UE[2] \\
\hline L11238 & GP5 glycoprotein V (platelet) & NS[1, 2] \\
\hline M36284 & GYPC glycophorin C (Gerbich blood group) & $\mathrm{NS}[1,2]$ \\
\hline M60750 & HIST1H2BG histone cluster $1, \mathrm{H} 2 \mathrm{bg}$ & $\mathrm{NS}[1,2]$ \\
\hline X79200 & Homo spaiens mRNA for SYT-SSX protein & $\mathrm{NS}[1,2]$ \\
\hline X92814 & HRASLS3 HRAS-like suppressor 3 & $\mathrm{NS}[1,2]$ \\
\hline M62831 & IER2 immediate early response 2 & $\mathrm{NS}[1,2]$ \\
\hline J04111 & JUN $^{*}$ v-jun sarcoma virus 17 oncogene homolog (avian) & $\mathrm{UE}[1,2]$ \\
\hline Y00503 & KRT19* keratin 19 & $\mathrm{NS}[1,2]$ \\
\hline X89430 & MECP2 methyl CpG binding protein 2 (Rett syndrome) & $\mathrm{NS}[1,2]$ \\
\hline U46499 & MGST1 microsomal glutathione S-transferase 1 & $\mathrm{NS}[1,2]$ \\
\hline M93221 & MRC1 mannose receptor, $C$ type 1 & $\mathrm{NS}[1,2]$ \\
\hline M76732 & MSX1* msh homeobox homolog 1 (Drosophila) & $\mathrm{NS}[1,2]$ \\
\hline S71824 & NCAM1 neural cell adhesion molecule 1 & $\mathrm{OE}[1], \mathrm{NS}[2]$ \\
\hline X70218 & PPP4C protein phosphatase 4 & NS $[1,2]$ \\
\hline U02680 & PTK9 protein tyrosine kinase 9 & $\mathrm{NS}[1,2]$ \\
\hline U79291 & PTPN11 protein tyrosine phosphatase, non-receptor type 11 & $\mathrm{NS}[1,2]$ \\
\hline
\end{tabular}


Table 2. Synapomorphies of Leiomyoma Specimens in Comparison to Normal Specimens (GDS533) (Cont'D)

\begin{tabular}{|c|c|c|}
\hline U77594 & $\begin{array}{l}\text { RARRES2* retinoic acid receptor responder (tazarotene } \\
\text { induced) } 2\end{array}$ & NS[1, 2] \\
\hline $\begin{array}{l}\text { M11433, } \\
\quad \text { X07438 }\end{array}$ & RBP1* retinol binding protein 1 , cellular & NS[1, 2] \\
\hline L20859 & $\begin{array}{l}\text { SLC20A1 solute carrier family } 20 \text { (phosphate transporter), } \\
\text { member } 1\end{array}$ & NS[1, 2] \\
\hline M97935 & $\begin{array}{l}\text { STAT1 signal transducer and activator of transcription } 1, \\
\text { 91kDa }\end{array}$ & NS[1, 2] \\
\hline J04152 & TACSTD2 tumor-associated calcium signal transducer 2 & NS[1, 2] \\
\hline X14787 & THBS1 thrombospondin 1 & $\mathrm{NS}[1,2]$ \\
\hline U24488 & $\mathrm{TNXB}^{*}$ tenascin $\mathrm{XB}$ & UE[1, 2] \\
\hline Z29083 & $\mathrm{TPBG}^{*}$ trophoblast glycoprotein & NS $[1,2]$ \\
\hline X51521 & VIL2 villin 2 (ezrin) & UE[1], NS[2] \\
\hline D87716 & WDR43 WD repeat domain 43 & NS[1, 2] \\
\hline \multicolumn{3}{|c|}{$\begin{array}{l}\text { C. Dichotomously-expressed synapomorphic genes: } \\
\text { ABCB1; ADRM1; AIM1; ALDH1A3; AMDD; ARHGEF6; ARL4D; ATP5B; Atp8a2; C1QB; CA9; CALM2; CTSB; } \\
\text { CCRL2; CD52; CD99; CPA3; DPYD; DSG2; Em×2; FEZ2; FLNA; FOXO1A; FYN; GAPDH; GNB3; GSTZ1; H1F0; H2- } \\
\text { ALPHA; HBG2; Ubx, Notch1; Hox5.4; HTR2C; ICA1; IGF2; INSR; ITGA6; ITGA9; KCNK1; KIAA0152; MAP1D; } \\
\text { MATK; MBP; MDM4; MFAP5; MFI2 antigen p97; MLH1; MPZ; NDUFS1; NELL2; NNAT; NOS3; NR4A1; OASL; } \\
\text { ODC1; OLFM1; PKN2; PON2; PRMT2; PSMC3; PTR2; RANBP2; RBMX; RDBP; RHOG; SAFB2; SCRIB; SELFP; } \\
\text { SERPINF1; SMS; SPOCK2; ST3GAL1; THRA; TNXB; TTLL4; TXNIP; UPK2; XA; ZNF43 }\end{array}$} \\
\hline
\end{tabular}

These comprise: 25 OE genes, 42 UE genes, and 79 DE genes. Asterisk $\left(^{*}\right)$ indicates a synapomorphy for leiomyosarcoma as well. Last column reports the status of the synapomorphies as described by [1] Hoffman et al. (2004) and [2] Quade et al. (2004) in their significant genes lists.

Table 3. A Clade of All Leiomyosarcoma Specimens Defined by 20 Synapomorphies in Comparison to Normal and Leiomyoma Specimens

A. Overexpressed synapomorphic genes:

X54942

U68566

CKS2 CDC28 proteininase regulatory subunit 2

HAX1 HCLS1 associated protein X-1

RDBP RD RNA binding protein

L03411

RRM1 ribonucleotide reductase M1 polypeptide

X59543

B. Underexpressed synapomorphic genes:

D13639

D21337

CCND2, cyclin D2

COL4A6 collagen, type IV, alpha 6

Csh2 chorionic somatomammotropin hormone 2

[Rattus norvegicus]

L36033

CXCL12 chemokine (C-X-C motif) ligand 12 (stromal cell-derived factor 1)

HG2663-HT2759_at

EMX2 empty spiracles homolog 2 (Drosophila).

Homeotic Protein Emx2

HG2663-HT2759_at

Homeotic Protein Emx2

HOXA10 homeobox A10 Expressed in the adult

human endometrium

AB002382

LOC284394 hypothetical gene supported by

NM_001331

U69263

U85707

MATN2 matrilin 2

Meis1, myeloid ecotropic viral integration site 1

homolog (mouse)

Z29678

L35240

MITF microphthalmia-associated transcription factor

PDLIM7 PDZ and LIM domain 7 (enigma)

D87735

RL14 ribosomal protein L14

L14076

SFRS4 splicing factor, arginine/serine-rich 4

SPTAN1 spectrin, alpha, nonerythrocytic 1 (alpha-

fodrin)

C. Dichotomously-expressed synapomorphic genes:

GAPDH glyceraldehyde-3-phosphate dehydrogenase 
Table 4. A Clade of All Leiomyosarcoma Specimens Defined by 29 Synapomorphies in Comparison to Leiomyoma Specimens Only (GDS533)

\begin{tabular}{|c|c|c|}
\hline \multicolumn{3}{|c|}{ A. Overexpressed synapomorphic genes: } \\
\hline X54941 & CKS1B CD28 protein kinase regulatory subunit 1B & $\mathrm{OE}$ \\
\hline X54942 & CKS2 CD28 protein kinase regulatory subunit 2 & NS \\
\hline J03060 & GBAP glucosidase, beta; acid, pseudogene & NS \\
\hline U78027 & $\begin{array}{l}\text { GLA galactosidase, alpha (associated w/Fabry's) RPL36A } \\
\text { ribosomal protein L36a }\end{array}$ & NS \\
\hline Y00433 & GPX1 glutathione peroxidase 1 & NS \\
\hline U68566 & HAX1 HCLS1 associated protein X-1 & NS \\
\hline X59543 & RRM1 ribonucleotide reductase M1 polypeptide & NS \\
\hline U12465 & RPL35 ribosomal protein L35 & $\mathrm{OE}$ \\
\hline U67674 & $\begin{array}{l}\text { SLC10A2 solute carrier family } 10 \text { (sodium/bile acid cotransporter } \\
\text { family), member } 2\end{array}$ & NS \\
\hline \multicolumn{3}{|c|}{ B. Underexpressed synapomorphic genes: } \\
\hline U87223 & CNTNAP1 contactin associated protein 1 & UE \\
\hline D30655 & EIF4A2 eukaryotic translation initiation factor $4 \mathrm{~A}$, isoform 2 & UE \\
\hline L20814 & GRIA2 glutamate receptor, ionotropic, AMPA 2 & UE \\
\hline M10051 & INSR insulin receptor & NS \\
\hline D79999 & LOC221181 hypothetical gene supported by NM_006437 & NS \\
\hline D14812 & MORF4L2 mortality factor 4 like 2 & UE \\
\hline L36151 & PIK4CA phosphatidylinositol 4-kinase, catalytic, alpha & NS \\
\hline D42108 & PLCL1 phospholipase C-like 1 & NS \\
\hline L13434 & RpL41 Ribosomal protein L41 & NS \\
\hline $\begin{array}{l}\text { HG921- } \\
\text { HT3995 }\end{array}$ & Serine/Threonine Kinase, Receptor 2-2, Alt. Splice 3 & NS \\
\hline D31891 & SETDB1 SET domain, bifurcated 1 & UE \\
\hline AB002318 & Talin2 & NS \\
\hline U53209 & TRA2A transformer-2 alpha & NS \\
\hline D87292 & TST thiosulfate sulfurtransferase (rhodanese) & NS \\
\hline M15990 & YES1 v-yes-1 Yamaguchi sarcoma viral oncogene homolog 1 & NS \\
\hline \multicolumn{3}{|c|}{ C. Dichotomously expressed synapomorphic genes: } \\
\hline U56417 & $\begin{array}{l}\text { AGPAT1 1-acylglycerol-3-phosphate O-acyltransferase } 1 \\
\text { (lysophosphatidic acid acyltransferase, alpha) }\end{array}$ & NS \\
\hline M63167 & AKT1 v-akt murine thymoma viral oncogene homolog 1 & NS \\
\hline L27560 & IGFBP5 insulin-like growth factor binding protein 5 & NS \\
\hline U40223 & P2RY4 pyrimidinergic receptor P2Y, G-protein coupled, 4 & NS \\
\hline D76444 & RNF103 ring finger protein 103 & NS \\
\hline
\end{tabular}

Last column reports the status of the synapomorphies as described by Quade et al. (2004) in their significant genes list

gram (Fig. 3). Its topology showed a total separation of the gastric cancer from the uterine leiomyoma and sarcoma specimens into two large clades. However, the two types of cancers shared 16 synapomorphies that delimited a clade composed of all the gastric and uterine specimens (Table 9).

The resulting inclusive cladogram (Fig. 3) showed an almost total agreement with the single type cladograms (Figs. 1 and 2), indicating a successful pooling of datasets. However, there was a slight variation in the topology of minor branches between the cladogram of Figure 2 and the inclusive one of Figure 3. These slight differences are most likely due to the increased number of normal specimens that were used in outgroup of the inclusive cladogram. Outgroup size used here was by no means ideal; the larger the membership of the outgroup the more stable the topology of the generated cladogram (Graybeal, 1998).

\section{Discussion}

Microarray data analysis aims to identify differentially expressed genes, and subsequently characterize genetic pat- terns, classify specimens accordingly, and point out potential biomarkers. However, most of the problems that are currently associated with microarray analysis arise from using only the quantitative aspect of the data (the absolute continuous data values of gene expression) to carry out parametric statistical analysis. Such a statistical analysis forecasts gene linkage on the basis of quantitative correlation and not expression pattern, and lacks the power to recognize and utilize specific gene expression patterns such as dichotomousexpression and partial asynchronicities (Abu-Asab et al., 2008; Allison et al., 2006). This results in discrepancies that affect which genes are considered differentially expressed by the two main ranking criteria for generating gene lists, the $t$-test and fold change (Guo 2006). Our phylogenetic analysis supports a qualitative approach where the directionality of expression is the first step to designate the expression value as significant, followed by parsimony search to plot a classification of specimens with the smallest number of steps that explains the data's distribution pattern.

This parsimonious analysis produced higher interplatform concordance than the gene lists generated with $t$-test 
Table 5. A Clade Composed of All Leiomyosarcoma Specimens is Defined in Relation to Normal Specimens (GDS533)

\begin{tabular}{|c|c|c|}
\hline \multicolumn{3}{|l|}{ A. Overexpressed } \\
\hline S78187 & CDC25B cell division cycle 25B & NS \\
\hline $\mathrm{U} 40343$ & $\begin{array}{l}\text { CDKN2D cyclin-dependent kinase inhibitor 2D (p19, } \\
\text { inhibits CDK4) }\end{array}$ & NS \\
\hline X54942 & CKS2 CDC28 protein kinase regulatory subunit 2 & NS \\
\hline X79353 & CDI1 GDP dissociation inhibitor 1 & NS \\
\hline X14850 & H2AFX H2A histone family, member $X$ & NS \\
\hline U51127 & IRF5 interferon regulatory factor 5 & NS \\
\hline U04209 & MFAP1 microfibrillar-associated protein 1 & NS \\
\hline U43177 & MpV17 mitochondrial inner membrane protein & NS \\
\hline U19796 & MRPL28 mitochondrial ribosomal protein L28 & $\mathrm{OE}$ \\
\hline $\mathrm{U} 37690$ & $\begin{array}{l}\text { POLR2L polymerase (RNA) II (DNA directed) } \\
\text { polypeptide L, } 7.6 \mathrm{kDa}\end{array}$ & NS \\
\hline M22960 & $\begin{array}{l}\text { PPGB protective protein for beta-galactosidase } \\
\text { (galactosialidosis) }\end{array}$ & NS \\
\hline U09210 & $\begin{array}{l}\text { SLC18A3 solute carrier family } 18 \text { (vesicular } \\
\text { acetylcholine), member } 3\end{array}$ & NS \\
\hline M86752 & $\begin{array}{l}\text { STIP1 stress-induced-phosphoprotein } 1 \text { (Hsp70/Hsp90- } \\
\text { organizing protein) }\end{array}$ & $\mathrm{OE}$ \\
\hline M26880 & UBC ubiquitin C & $\mathrm{OE}$ \\
\hline U43177 & UCN urocortin & NS \\
\hline \multicolumn{3}{|c|}{ B. Underexpressed synapomorphic genes: } \\
\hline M12963 & $\begin{array}{l}\text { ADH1A alcohol dehydrogenase 1A (class I), alpha } \\
\text { polypeptide }\end{array}$ & UE \\
\hline $\begin{array}{l}\text { HG3638- } \\
\text { HT3849_s_at }\end{array}$ & $\begin{array}{l}\text { Amyloid Beta (A4) Precursor Protein, Alt. Splice 2, } \\
\text { A4(751) }\end{array}$ & NS \\
\hline L28997 & ARL1 ADP-ribosylation factor-like 1 & NS \\
\hline Z49269 & CCL14 chemokine (C-C motif) ligand 14 & UE \\
\hline M92934 & CTGF connective tissue growth factor & UE \\
\hline M74099 & $\begin{array}{l}\text { CUTL1 cut-like 1, CCAAT displacement protein } \\
\text { (Drosophila) }\end{array}$ & NS \\
\hline M96859 & DPP6 dipeptidyl-peptidase 6 & UE \\
\hline U94855 & $\begin{array}{l}\text { EIF3S5 eukaryotic translation initiation factor } 3 \text {, subunit } 5 \\
\text { epsilon, } 47 \mathrm{kDa}\end{array}$ & NS \\
\hline L25878 & EPHX1 epoxide hydrolase 1, microsomal (xenobiotic) & NS \\
\hline $\begin{array}{l}\text { U60061- } \\
\text { U69140 }\end{array}$ & FEZ2 fasciculation and elongation protein zeta 2 (zygin II) & NS \\
\hline X67491 & GLUDP5 glutamate dehydrogenase pseudogene 5 & NS \\
\hline $\begin{array}{l}\text { HG4334- } \\
\text { HT4604_s_at }\end{array}$ & Glycogenin & NS \\
\hline X53296 & IL1RN interleukin 1 receptor antagonist & NS \\
\hline X55740 & NT53 5'-nucleotidase, ecto (CD73) & UE \\
\hline X78136 & PCBP2 poly $(\mathrm{rC})$ binding protein 2 & UE \\
\hline Z50194 & $\begin{array}{l}\text { PHLDA1 pleckstrin homology-like domain, family A, } \\
\text { member } 1\end{array}$ & NS \\
\hline J02902 & $\begin{array}{l}\text { PPP2R1A protein phosphatase } 2 \text { (formerly 2A), regulatory } \\
\text { subunit A (PR 65), alpha isoform }\end{array}$ & NS \\
\hline J03805 & $\begin{array}{l}\text { PPP2CB protein phosphatase 2, catalytic subunit, beta } \\
\text { isoform }\end{array}$ & NS \\
\hline U25988 & PSG11 pregnancy specific beta-1-glycoprotein 11 & NS \\
\hline M98539 & PTGDS prostaglandin D2 synthase $21 \mathrm{kDa}$ (brain) & UE \\
\hline X54131 & PTPRB protein tyrosine phosphatase, receptor type, B & NS \\
\hline M12174 & RHOB ras homolog gene family, member B & NS \\
\hline $\begin{array}{l}\text { HG1879- } \\
\text { HT3521_at }\end{array}$ & RHOQ ras homolog gene family, member $\mathrm{Q}$ & NS \\
\hline X98534 & VASP vasodilator-stimulated phosphoprotein & NS \\
\hline X51630 & WT1 Wilms tumor 1 & UE \\
\hline $\begin{array}{l}\text { HG3426- } \\
\text { HT3610 s at }\end{array}$ & Zinc Finger Protein Hzf-16, Kruppel-Like, Alt. Splice 1 & NS \\
\hline M92843 & ZFP36 zinc finger protein $36, \mathrm{C} 3 \mathrm{H}$ type, homolog (mouse) & UE \\
\hline \multicolumn{3}{|c|}{ C. Dichotomously expressed synapomorphic genes: } \\
\hline U80226 & ABAT 4-aminobutyrate aminotransferase & NS \\
\hline M14758 & $\begin{array}{l}\text { ABCB1 ATP-binding cassette, sub-family B (MDR/TAP), } \\
\text { member } 1\end{array}$ & NS \\
\hline M95178 & ACTN1 actinin, alpha 1 & $\begin{array}{r}\text { NS } \\
\text { ued) }\end{array}$ \\
\hline
\end{tabular}


Table 5. A Clade Composed of All Leiomyosarcoma Specimens is Defined in Relation to Normal Specimens (GDS533) (Cont'D)

\begin{tabular}{|c|c|c|}
\hline U76421 & $\begin{array}{l}\text { ADARB12 adenosine deaminase, RNA-specific, B1 (RED1 } \\
\text { homolog rat) }\end{array}$ & NS \\
\hline U46689 & ALDH3A2 aldehyde dehydrogenase 3 family, member A2 & NS \\
\hline L34820 & $\begin{array}{l}\text { ALDH5A1 aldehyde dehydrogenase } 5 \text { family, member A1 } \\
\text { (succinate-semialdehyde dehydrogenase) }\end{array}$ & NS \\
\hline M84332 & ARF1 ADP-ribosylation factor 1 & NS \\
\hline D14710 & $\begin{array}{l}\text { ATP5A1 ATP synthase, } \mathrm{H}+\text { transporting, mitochondrial F1 } \\
\text { complex, alpha subunit } 1 \text {, cardiac muscle }\end{array}$ & NS \\
\hline X84213 & BAK1 BCL2-antagonist/killer 1 & NS \\
\hline U23070 & $\begin{array}{l}\text { BAMBI BMP and activin membrane-bound inhibitor } \\
\text { homolog (Xenopus laevis) }\end{array}$ & NS \\
\hline M33518 & BAT2 HLA-B associated transcript 2 & NS \\
\hline X61123 & BTG1 B-cell translocation gene 1, anti-proliferative & NS \\
\hline S60415 & CACNB2 calcium channel, voltage-dependent, beta 2 subunit & NS \\
\hline M19878 & CALB1 calbindin 1, $28 \mathrm{kDa}$ & NS \\
\hline L76380 & CALCRL calcitonin receptor-like & NS \\
\hline M21121 & CCL5 chemokine ( $\mathrm{C}-\mathrm{C}$ motif) ligand 5 & NS \\
\hline D14664 & CD302 CD302 molecule & NS \\
\hline X72964 & CETN2 centrin, EF-hand protein, 2 & NS \\
\hline U66468 & CGREF1 cell growth regulator with EF-hand domain 1 & NS \\
\hline M63379 & CLU clusterin & NS \\
\hline X52022 & COL6A3 collagen, type VI, alpha 3 & UE \\
\hline L25286 & COL15A1 collagen, type XV, alpha 1 & NS \\
\hline S45630 & CRYAB crystallin, alpha B & NS \\
\hline X95325 & CSDA cold shock domain protein A & NS \\
\hline U03100 & $\begin{array}{l}\text { CTNNA1 catenin (cadherin-associated protein), alpha } 1 \text {, } \\
\text { 102kDa }\end{array}$ & NS \\
\hline X52142 & CTPS CTP synthase & NS \\
\hline D38549 & CYFIP1 cytoplasmic FMR1 interacting protein 1 & NS \\
\hline X64229 & DEK DEK oncogene (DNA binding) & NS \\
\hline M63391 & DES desmin & UE \\
\hline Z34918 & EIF4G3 eukaryotic translation initiation factor 4 gamma, 3 & NS \\
\hline U97018 & EML1 echinoderm microtubule associated protein like 1 & NS \\
\hline U12255 & FCGRT Fc fragment of IgG, receptor, transporter, alpha & NS \\
\hline U36922 & FOXO1A forkhead box O1A (rhabdomyosarcoma) & NS \\
\hline U91903 & FRZB frizzled-related protein & NS \\
\hline M33197 & GAPDH glyceraldehyde-3-phosphate dehydrogenase & NS \\
\hline U09587 & GARS glycyl-tRNA synthetase & NS \\
\hline U66075 & GATA6 GATA binding protein 6 & NS \\
\hline D13988 & GDI2 GDP dissociation inhibitor 2 & NS \\
\hline $\mathrm{U} 31176$ & $\begin{array}{l}\text { GFER growth factor, augmenter of liver regeneration } \\
\text { (ERV1 homolog, S. cerevisiae) }\end{array}$ & NS \\
\hline U28811 & GLG1 golgi apparatus protein 1 & NS \\
\hline $\mathrm{U} 66578$ & GPR23 G protein-coupled receptor 23 & NS \\
\hline L40027 & GSK3A glycogen synthase kinase 3 alpha & NS \\
\hline U77948 & GTF2I general transcription factor II, $\mathrm{i}$ & UE \\
\hline Z29481 & HAAO 3-hydroxyanthranilate 3,4-dioxygenase & NS \\
\hline D16480 & $\begin{array}{l}\text { HADHA hydroxyacyl-Coenzyme A dehydrogenase/3- } \\
\text { ketoacyl-Coenzyme A thiolase/enoyl-Coenzyme A } \\
\text { hydratase (trifunctional protein), alpha subunit }\end{array}$ & NS \\
\hline U50079 & HDAC1 histone deacetylase 1 & NS \\
\hline U50078 & $\begin{array}{l}\text { HERC1 hect (homologous to the E6-AP (UBE3A) carboxyl } \\
\text { terminus) domain and RCC1 (CHC1)-like domain (RLD) } 1\end{array}$ & NS \\
\hline M95623 & HMBS hydroxymethylbilane synthase & NS \\
\hline X79536 & HNRPA1 heterogeneous nuclear ribonucleoprotein A1 & NS \\
\hline L15189 & HSPA9B heat shock 70 kDa protein 9B (mortalin-2) & NS \\
\hline U05875 & $\begin{array}{l}\text { IFNGR2 interferon gamma receptor } 2 \text { (interferon gamma } \\
\text { transducer 1) }\end{array}$ & NS \\
\hline X57025 & IGF1 insulin-like growth factor 1 (somatomedin C) & UE \\
\hline HG3543- & IGF2 insulin-like growth factor 2 (somatomedin A) & NS \\
\hline $\mathrm{U} 40282$ & ILK integrin-linked kinase & NS \\
\hline X74295 & ITGA7 integrin, alpha 7 & NS \\
\hline X57206 & ITPKB inositol 1,4,5-trisphosphate 3-kinase B & NS \\
\hline AB002365 & KIAA0367 & UE \\
\hline
\end{tabular}


Table 5. A Clade Composed of All Leiomyosarcoma Specimens is Defined in Relation to Normal Specimens (GDS533) (CONT'D)

\begin{tabular}{|c|c|c|}
\hline J00124 & $\begin{array}{l}\text { KRT14 keratin } 14 \text { (epidermolysis bullosa simplex, } \\
\text { Dowling-Meara, Koebner) }\end{array}$ & NS \\
\hline X05153 & LALBA lactalbumin, alpha- & NS \\
\hline X02152 & LDHA lactate dehydrogenase A & NS \\
\hline $\begin{array}{l}\text { HG3527- } \\
\text { HT3721_f_at }\end{array}$ & LHB luteinizing hormone beta polypeptide & NS \\
\hline X86018 & LRRC41 leucine rich repeat containing 41 & NS \\
\hline L38486 & MFAP4 microfibrillar-associated protein 4 & NS \\
\hline D87742 & MIA3 melanoma inhibitory activity family, member 3 & NS \\
\hline M69066 & MSN moesin & NS \\
\hline AB003177 & mRNA for proteasome subunit p27 & NS \\
\hline $\mathrm{U} 47742$ & $\begin{array}{l}\text { MYST3 MYST histone acetyltransferase (monocytic } \\
\text { leukemia) } 3\end{array}$ & NS \\
\hline M30269 & NID1 nidogen 1 & NS \\
\hline U80669 & NKX3-1 NK3 transcription factor related, locus 1 (Drosophila) & NS \\
\hline M10901 & $\begin{array}{l}\text { NR3C1 nuclear receptor subfamily } 3 \text {, group C, member } 1 \\
\text { (glucocorticoid receptor) }\end{array}$ & \\
\hline M16801 & NR3C2 nuclear receptor subfamily 3, group C, member 2 & NS \\
\hline U52969 & PCP4 Purkinje cell protein 4 & UE \\
\hline J03278 & $\begin{array}{l}\text { PDGFRB platelet-derived growth factor receptor, beta } \\
\text { polypeptide }\end{array}$ & NS \\
\hline D37965 & PDGFRL platelet-derived growth factor receptor-like & NS \\
\hline Z49835 & PDIA3 protein disulfide isomerase family $\mathrm{A}$, member 3 & NS \\
\hline U78524 & PIAS1 protein inhibitor of activated STAT, 1 & NS \\
\hline U60644 & PLD3 phospholipase D family, member 3 & NS \\
\hline D11428 & PMP22 peripheral myelin protein 22 & NS \\
\hline U79294 & PPAP2B phosphatidic acid phosphatase type $2 \mathrm{~B}$ & NS \\
\hline S71018 & PPIC peptidylprolyl isomerase C (cyclophilin C) & NS \\
\hline X07767 & PRKACA protein kinase, cAMP-dependent, catalytic, alpha & NS \\
\hline X83416 & PRNP prion protein $(\mathrm{p} 27-30)$ & NS \\
\hline M555671 & PROZ protein Z, vitamin K-dependent plasma glycoprotein & NS \\
\hline U72066 & RBBP8 retinoblstoma binding protein $8^{1}$ & NS \\
\hline L25081 & RHOC ras homolog gene family, member $C$ & NS \\
\hline U40369 & SAT1 spermidine/spermine N1-acetyltransferase 1 & NS \\
\hline M97287 & $\begin{array}{l}\text { SATB1 special AT-rich sequence binding protein } 1 \text { (binds } \\
\text { to nuclear matrix/scaffold-associating DNAs) }\end{array}$ & \\
\hline U83463 & SDCBP syndecan binding protein (syntenin) & NS \\
\hline U28369 & $\begin{array}{l}\text { SEMA3B sema domain, immunoglobulin domain (Ig), } \\
\text { short basic domain, secreted, (semaphorin) 3B }\end{array}$ & \\
\hline $\begin{array}{l}\text { HG3925- } \\
\text { ht4195_at }\end{array}$ & SFTPA2 surfactant, pulmonary-associated protein A2 & NS \\
\hline L31801 & $\begin{array}{l}\text { SLC16A1 solute carrier family } 16, \text { member } 1 \\
\text { (monocarboxylic acid transporter } 1 \text { ) }\end{array}$ & \\
\hline M91463 & $\begin{array}{l}\text { SLC2A4 solute carrier family } 2 \text { (facilitated glucose } \\
\text { transporter), member } 4\end{array}$ & \\
\hline U66617 & $\begin{array}{l}\text { SMARCD1 SWI/SNF related, matrix associated, actin } \\
\text { dependent regulator of chromatin, subfamily } \mathrm{d} \text {, member } 1\end{array}$ & \\
\hline U50383 & SMYD5 SMYD family member 5 & NS \\
\hline D43636 & SNRK SNF related kinase & NS \\
\hline D87465 & $\begin{array}{l}\text { SPOCK2 sparc/osteonectin, cwcv and kazal-like domains } \\
\text { proteoglycan (testican) } 2\end{array}$ & \\
\hline M61199 & SSFA2 sperm specific antigen 2 & NS \\
\hline U15131 & ST5 suppression of tumorigenicity 5 & NS \\
\hline U95006 & STRA13 stimulated by retinoic acid 13 homolog (mouse) & NS \\
\hline M74719 & TCF4 transcription factor 4 & NS \\
\hline X14253 & TDGF1 teratocarcinoma-derived growth factor 1 & NS \\
\hline U52830 & TERT telomerase reverse transcriptase & NS \\
\hline U12471 & THBS1 thrombospondin 1 & NS \\
\hline U16296 & TIAM1 T-cell lymphoma invasion and metastasis 1 & JS \\
\hline L01042 & TMF1 TATA element modulatory factor 1 & JS \\
\hline U03397 & $\begin{array}{l}\text { TNFRSF9 tumor necrosis factor receptor subfamily, } \\
\text { member } 9\end{array}$ & \\
\hline
\end{tabular}


Table 5. A Clade Composed of All Leiomyosarcoma Specimens is Defined in Relation to Normal Specimens (GDS533) (CONT’D)

\begin{tabular}{lll}
\hline X05276 & TMP4 tropomyosin 4 & \\
HG4683- & TRAF2 TNF receptor-associated factor 2 & UE \\
HT5108_s_at & UFD1L ubiquitin fusion degradation 1 like (yeast) & NS \\
U64444 & UBE2D3 ubiquitin-conjugating enzyme E2D 3 (UBC4/5 & NS \\
U39318 & homolog, yeast) & NS \\
X59739 & ZFX zinc-finger protein, X-linked & \\
\hline
\end{tabular}

Last column reports the status of the synapomorphies as described by Quade et al. (2004) in their significant genes list

and fold change (Tables 7 and 8), and allowed the pooling and comparability of two independent experiments. Such results confer reliability to a qualitative parsimonious approach to analyzing gene expression data. Table 10 summarizes the major characteristics of a parsimony phylogenetic approach.
In addition to its evident scientific applications, a phylogenetic analysis as outlined here has clinical implications as well. Indeed, the results of the three microarray gene expression datasets show the clinical potential for such parsimonious analysis; they produced a total distinction of the sarcoma from the fibroid tissues (the leiomyomas), and these

Table 6. A List of 34 Synapomorphies Defining a Clade Composed of All Gastric Cancer Specimens (GDS1210)

\begin{tabular}{|c|c|c|}
\hline \multicolumn{3}{|c|}{ A. Overexpressed synapomorphic genes: } \\
\hline X81817 & BAP31 mRNA & No \\
\hline D50914 & BOP1 block of proliferation 1 & No \\
\hline X54667 & CST4: cystatin S MGC71923 & Yes \\
\hline L17131 & HMGA1 high mobility group AT-hook 1 & No \\
\hline D63874 & HMGB1 high-mobility group box 1 & No \\
\hline D26600 & PSMB4 proteasome (prosome, macropain) subunit, beta type, 4 & No \\
\hline U36759 & PTCRA pre-T-cell antigen receptor alpha PT-ALPHA, PTA & No \\
\hline X89750 & TGIF TGFB-induced factor (TALE family homeobox) & No \\
\hline \multicolumn{3}{|c|}{ B. Underexpressed synapomorphic genes: } \\
\hline X76342 & ADH7 alcohol dehydrogenase 7 (class IV), mu or sigma polypeptide ADH-4 & No \\
\hline M63962 & ATP4A ATPase, $\mathrm{H}+/ \mathrm{K}+$ exchanging, alpha polypeptide ATP6A & No \\
\hline M75110 & ATP4B ATPase, $\mathrm{H}+/ \mathrm{K}+$ exchanging, beta polypeptide ATP6B & No \\
\hline J05401 & CKMT2 creatine kinase, mitochondrial 2 (sarcomeric) & No \\
\hline L38025 & DNTFR ciliary neurotrophic factor receptor & No \\
\hline M61855 & CYP2C9: cytochrome P450, family 2 , subfamily C, polypeptide 9 CPC9 & No \\
\hline D63479 & DGKD: diacylglycerol kinase, delta 130kDa DGKdelta, KIAA0145, dgkd-2 & No \\
\hline X99101 & ESR2 estrogen receptor 2 (ER beta) & No \\
\hline U21931 & FBP1 fructose-1, 6-bisphosphatase 1 & No \\
\hline $\begin{array}{l}\text { HG3432- } \\
\text { HT3618 a }\end{array}$ & Fibroblast Growth Factor Receptor K-Sam, Alt. Splice 1 & No \\
\hline M31328 & GNB3 guanine nucleotide binding protein (G protein), beta polypeptide 3 & No \\
\hline D42047 & GPD1L glycerol-3-phosphate dehydrogenase 1-like & No \\
\hline M62628 & Human alpha-1 Ig germline C-region membrane-coding region, 3'end & No \\
\hline D29675 & Human inducible nitric oxide synthase gene, promoter and exon 1 & No \\
\hline M63154 & Human intrinsic factor mRNA & No \\
\hline Z29074 & KRT9 keratin 9 (epidermolytic palmoplantar keratoderma) EPPK, K9 & No \\
\hline X05997 & LIPF lipase, gastric & No \\
\hline U50136 & LTC4S leukotriene C4 synthase MGC33147 & No \\
\hline X76223 & MAL: mal, T-cell differentiation protein & No \\
\hline U19948 & PDIA2 protein disulfide isomerase family A, member 2 & No \\
\hline L07592 & PPARD peroxisome proliferative activated receptor, delta & No \\
\hline U57094 & RAB27A, member RAS oncogene family & No \\
\hline AC002077 & SLC 38A3 solute carrier family 38 , member 3 & No \\
\hline $\mathrm{Z} 29574$ & TNFRSF17 tumor necrosis factor recepter superfamily, member 17 & No \\
\hline \multicolumn{3}{|c|}{ C. Dichotomously expressed synapomorphic genes: } \\
\hline D00408 & $\begin{array}{l}\text { CYP3A7 cytochrome P450, family } 3 \text {, subfamily A, polypeptide } 7 \text { CP37, } \\
\text { P450-HFLA }\end{array}$ & No \\
\hline U29092 & SELENBP1 selenium binding protein 1 & No \\
\hline
\end{tabular}

Synapomorphies include: 8 OE genes, 24 UE genes, and 2 DE genes in comparison with the normal specimens. Last column reports the status of the synapomorphies as described by Hippo et al. (2002). Yes $=$ listed; No $=$ not listed. 
TABle 7. INTERPLATFORM CONCORDANCE

GDS533

GDS484

A. Overexpressed synapomorphic genes:

a. Identical Synapomorphies

DDB2

FUT8

MEST

TMSL8

TYMS

b. Homologous synapomorphies

CACNB3

COL5A2

KIAA0367

PRKAR1B

B. Underexpressed synapomorphic genes:

a. Identical synapomorphies

ALDH1A1

ALDH2

ATF3

CEBPD

CXADR

CYR61

DUSP1

FOS

HRASLS3

IER2

JUN

KRT19

RARRES2

TACSTD2

TNXB

VIL2

b. Homologous synapomorphies

CASP9

CYBA

FOSB

JUNB

PPP4C

SLC20A1

THBS1

WDR43

C. Dichotomously expressed synapomorphic genes:

a. Identical synapomorphies

CTSB

b. Homologous synapomorphies

ARL4D

FOXO1A

GNB3

ITGA6

ITGA9

KCNK1

MFAP5

PSMC3

SELP

TXNIP

ZNF43

CTSB

ALDH1A1

ALDH2

ATF3

CEBPD

CXADR

CYR61

DUSP1

FOS

HRASLS3

IER2

JUN

KRT19

RARRES2

TACSTD2

TNXB

VIL2

CASP4

CYB5R1

FOS

JUN

PPP1R10

SLC18A2

THBD

WDR37

ARL4C

FOXJ3

GNB1L

ITGA2B

ITGA2B

KCNJ5

MFAP4

PSMC2

SELL

TXNDC13

ZNF259P

A list of overlapping identical (22) and homologous (23) synapomorphic genes in leiomyoma specimens of GDS484 and GDS533. These include: $9 \mathrm{OE}, 24 \mathrm{UE}$, and $12 \mathrm{DE}$.

two classes from gastric cancer. It also identified a number of synapomorphies for gastric and uterine cancers, thus defining each as a separate disease entity with its unique shared derived expressions (see also Meza-Zepeda et al.
2006, for further support of this point). Furthermore, the combined analysis revealed the shared alterations of gene expression that are shared between the uterine and gastric cancers (Table 9). This conclusion is supported by the presence of these synapomorphic gene expressions as significant ones in other types of cancers as well: bladder, breast, colorectal, ovarian, pancreatic, prostate, and renal (GuzińskaUstymowicz et al., 2008; Dong et al., 2007; Huang et al., 2006; Pilarsky et al., 2004).

\section{Advantages of polarity assessment}

There are several reasons for our preference of a combination of polarity assessment via outgroup comparison and parsimony over other methods for the analysis of gene expression microarray data, an approach that is also supported by other authors (Allison et al., 2006; Kolaczkowski and Thornton, 2004). Parsimony phylogenetic analysis requires polarity assessment for each data value to determine its novelty-whether it represents a change from the normal state (Abu-Asab et al., 2008). We advocate that qualitative, and not only quantitative, similarity is a better measure of common ontogenetic steps among specimens, and that a correlation of genes based on similar quantitative expression is not necessarily indicative of ontogenic relationships among genes.

Polarity assessment does not set an arbitrary stringency on gene selection, especially where the distribution pattern is gene specific within a set of specimens (e.g., DE and partially asynchronous genes), while other methods are not optimal for its assessment (Huang and Qu, 2006; Lyons-Weiler et al., 2004). Fold change and $F$ and $t$-statistics may dismiss from the gene list those genes with dichotomous expressions, although they are indicative of a unique expression type and may account for some phenomena such as transitional clades located between diseased and normal clades, and multiple developmental pathways in some disease types (Abu et al., 2006; Lyons-Weiler et al., 2004). The gene lists of Tables $1 \mathrm{C}-7 \mathrm{C}$ show a large number of DE asynchronous genes that were mostly not considered significant by other methods (Hippo et al., 2002; Hoffman et al., 2004; Quade et al., 2004), or their dichotomous mode was not noticed by the authors.

Because polarity assessment transforms the quantitative data into a qualitative matrix, it reduces the data noise. The absolute quantitative nature of the microarray data restricts their use and interpretation due to their range of inconsistencies between runs, platforms, and laboratories. By polarizing each data set with its own set of outgroup specimens, the inconsistencies of the experiment are eliminated since the polarization process is a comparison between equals-data values generated at the same time. The benefit here translates into the ability to pool a large number of experiments, carry out intra- and interplatform comparabilities, and a better gene list concordance between experiments. However, as discussed below, polarity assessment is sensitive to the choice and size of the outgroup specimens.

\section{Selection and size of the outgroup}

When conducting a polarity assessment, outgroup's selection and its effective size are very significant factors in correctly identifying synapomorphies, and therefore, optimally delimiting the classes of diseased specimens. The 
Table 8. Summary of CONCORDance Results betweEn GDS484 and GDS533

\begin{tabular}{|c|c|c|c|c|}
\hline & & \multicolumn{3}{|c|}{ GDS533 (Fibroids and Leiomyosarcomas) } \\
\hline & & $\begin{array}{l}\text { Quad et al. } \\
\text { Gene List }\end{array}$ & $\begin{array}{l}\text { Abu-Asab et al. } \\
\text { Synapomorphies } \\
\text { for Fibroids (146) }\end{array}$ & $\begin{array}{c}\text { Abu-Asab et al. } \\
\text { Synapomorphies for } \\
\text { Fibroids and } \\
\text { Leiomyosarcoma } \\
\text { (32) }\end{array}$ \\
\hline \multirow[t]{2}{*}{$\begin{array}{l}\text { GDS484 } \\
\text { (Fibroids) }\end{array}$} & $\begin{array}{c}\text { Hoffman et al. } \\
\text { Gene List }\end{array}$ & $12 \%$ & $18 \%$ & $19.3 \%$ \\
\hline & $\begin{array}{l}\text { Abu-Asab et al. } \\
\text { Synapomorphies } \\
\text { for Fibroids } \\
(1485)\end{array}$ & $20 \%$ & $31 \%$ & $48 \%$ \\
\hline \multicolumn{2}{|c|}{ GDS533 Quad et al. Gene List } & $\mathrm{N} / \mathrm{A}$ & $16.5 \%$ & $45 \%$ \\
\hline
\end{tabular}

The comparisons were carried out in various combinations: statistical versus statistical, phylogenetic versus statistical, and phylogenetic versus phylogenetic. N/A: not applicable (number of synapomorphies).

composition of the outgroup specimens affects the outcome of the analysis as demonstrated by the different combinations of outgroups that we used to conduct polarity assessment for the leiomyosarcomas and leiomyomas (Tables 1-5). We selected these various arrangements of outgroups to demonstrate that their compositions produced slightly different but still biologically meaningful results. It is important to note that the outgroup should be composed of only healthy specimens when the goal is to find out the genes involved in disease inception, progression, and maintenance. As Tables 1-5 show, variations of out/ingroup composition lead to variations in identifying synapomorphies. Also, Figure 3 shows that the different types of cancers will separate into their respective clades when the outgroup is composed of normal specimens; a process that can be utilized for diagnosis.

In our combined analysis (Fig. 3), the increase in outgroup size did not affect the major topology of the cladogram, but rather the internal branching of some clades (normal and gastric cancer) when compared with their single analysis (Figs. 1-2). Because increasing the number of genes in the study does not have the same effect as enlarging outgroup size (Graybeal, 1998), it is our conclusion that a successful analysis requires a good number of normal specimens to be used as the outgroup. For microarray experiments to be meaningful and provide high predictivity, the smallest number of normal specimens that encompasses the maximum variation per population should be established and used in the analysis.

\section{Inferring gene linkage through parsimony phylogenetic analysis}

Whereas gene linkage of a clustering dendrogram is based on quantitative correlations between differentially expressed

TABle 9. INTERPLATFORM COMPARABILITy

\begin{tabular}{ll}
\hline ID & \\
\hline U52522 & Gene \\
U51478 & ATP1B3 ATPase, NaK transporting, beta 3 polypeptide \\
X66839 & CA9 carbonic anhydrase IX \\
M60974 & GADD45A growth arrest and DNA-damage-inducible, alpha \\
X01677- & GAPDH glyceraldehyde-3-phosphate dehydrogenase [two \\
M33197 & readings] \\
X14850 & H2AFX H2A histone family, member X \\
U52830 & Homo sapiens Cri-du-chat region mRNA, clone CSC8 \\
U25138 & KCNMB1 potassium large conductance calcium-activated \\
& channel, subfamily M, beta member 1 \\
D21063 & MCM2 minichromosome maintenance deficient 2 \\
L38486 & MFAP4 microfibrillar-associated protein 4 \\
D87463 & PHYHIP phytanoyl-CoA 2-hydroxylase interacting protein \\
X02419 & PLAU plasminogen activator, urokinase \\
L48513 & PON2 paraoxonase 2 \\
U29091 & SELENBP1 selenium binding protein 1 \\
Z19083 & TPBG trophoblast glycoprotein \\
M25077 & TROVE2 TROVE domain family, member 2 \\
\hline
\end{tabular}

A list of 16 synapomorphies defining a clade composed of all gastric cancer (GDS1210) as well as uterine sarcoma and leiomyom specimens (GDS533). 
Table 10. Summary of the Characteristics of a Parsimonious Phylogenetic Analysis through Polarity Assessment of Gene-Expression Values Followed by a MaXimum Parsimony ANALysis

- Offers a qualitative assessment of microarray gene expression data by sorting expression values into derived or ancestral.

- Identifies synapomorphies (shared derived expression states) and uses them to delineate clades (class discovery). Synapomorphies are also the potential biomarkers.

- Searches for the most parsimonious classification of specimens; the one with minimum number of steps, reversals, and parallels.

- Efficiently models the heterogeneous expression profiles of the diseased specimens. Those with fast mutation rate such as cancer.

- Incorporates gene expressions that violate normal distribution in a set of specimens-e.g., dichotomously expressed genes.

- Reduces the sensitivity to experimental noise.

- Permits pooling of multiple experiments.

- Allows intra and intercomparability of data.

- Produces higher concordance between gene lists than statistical methods ( $F \& t$-statistics and fold change).

- Offers a nonparametric data-based, not specimen-based, gene listing and gene linkage.

genes, in a parsimony cladogram it is based on the distribution of derived and ancestral gene expression states of all genes of all the specimens; that is, it is a map of expression states-both ancestral and derived. It reflects the classification that has the lowest number of steps as well as parallels and reversals to explain the distribution of expression states among specimens.

Gene linkage here is based on the location of genes on the cladogram. The synapomorphies below a node on the cladogram are the linked genes that are shared among the specimens above that node. Because a parsimonious cladogram is hierarchical, every one of its nodes has its synapomorphy(ies). This characteristic of a cladogram presents it as a map of linked genetic alterations that produce the diversity/relatedness of its specimens and may also permit the tracing of shared ontogenic pathways that are responsible for disease initiation and progression.

\section{Improved interplatform concordance and comparability}

Improved interplatform concordance is a criterion that will confer robustness and significance on microarray as a valid experimental and clinical platform. Our tests of concordance by comparing the lists of synapomorphies generated by polarity assessment of two experiments produced better results than those of fold change and F-statistic, and better than between the latter two (Table 8). When comparing the synapomorphies of a clade composed of leiomyomas and leiomyosarcomas (GDS533) with the synapomorphies of leiomyomas (GDS484), we obtained a high concordance of $89 \%$ within over- and underexpressed and 35\% within dichotomously expressed genes. The concordance between the two studies could have been higher if the number of probes of the GDS533 was closer to GDS484-7,000 versus 22,000
(Hoffman et al., 2004; Quade et al., 2004). Furthermore, even a comparison of the synapomorphies of two leiomyoma groups [GDS484 (1485 synapomorphies) and GDS533 (146 synapomorphies; Table 2)] produced 31\% concordance between the two groups of leiomyoma (45/146; Table 7). Nevertheless, this was a higher percentage than was produced by statistical methods (12\%).

Interplatform comparability has been difficult to carry out on microarray data because of data inconsistencies between runs, experiments, and laboratories; however, with polarity assessment, which converts the quantitative values of gene expression of every experiment into a qualitative matrix, it is possible to combine several matrices and carry out intraand interplatform comparisons in a parsimonious phylogenetic sense. A phylogenetic interplatform comparability of microarray data can be carried out if each dataset can be polarized separately with its own outgroup to produce its polarized matrix. Furthermore, when their probes are identical, two or more polarized sets can be pooled together and analyzed as Figure 3 shows. We have successfully pooled and analyzed two separately polarized datasets (GDS533 and 1210) of gastric cancer as well as uterine leiomyoma and leiomyosarcoma, where the two datasets were prepared separately but on an identical gene chip platform, GPL80.

\section{Implications on disease definition, profiling, diagnosis, and prognosis}

Although it is assumed that each disease has its own unique developmental pathway(s) (Adsay et al., 2002; Chung, 2000; Hayashi et al., 2004), thus far, the omics data has not been used to prove this premise. Our analysis of two independently generated datasets that represent uterine (GDS533) and gastric (GDS1210) cancers confirms that each of these two types of cancer is a natural class of specimens (a clade) that is circumscribed by its own set of synapomorphies. If this can be extended to other types of cancer, then each cancer can be considered a natural clade with its unique gene expression identifiers-the synapomorphies.

There are several implications to this conclusion; the most obvious is its effect on the definition of biomarkers. If the specimens of a type of cancer form a clade, then any suggested biomarker has to be selected from the clades' synapomorphies; otherwise, it will not be a universal diagnostic test for all the specimens of this cancer. Some of the currently applied immunohistomarkers are not universal synapomorphies. For example, the memberships of all four clades of the gastric cancers (Fig. 2) did not correlate well with the specimens' immunoreactivity to antibodies against p53, E-cadherin, and $\beta$-catenin, and a published two-way clustering did not correlate any better (Hippo et al., 2002). The discordance between omics biomarkers and most of the currently used immunohistological markers is a problem that can be better addressed in a phylogenetic sense. The discordance between microarray and immunohistochemical (IHC) biomarkers is due to the differing natures of these two types of markers. Although gene-expression biomarkers are based on the averaging of the expression values of the number of the cells used in the study (i.e., the cell homogenate), the IHC is based on the pathologist reading of the percentage of positive cells in the stained section. The IHC result is given as a percentage figure that could start at $10 \%$, and is used to associate 
the neoplasm with the normal tissue of origin. For an accurate and meaningful interpretation of the gene expression analyses, a comparison between the two types of biomarkers should be avoided, and the phylogenetic concept that is suggested here should be adopted.

A second implication is that a phylogenetic classification can be a clinical tool to carry out early detection, diagnosis, grading, prognosis, and posttreatment evaluation; these tasks can be realized through a parsimony analysis where the place of a specimen within the cladogram (i.e., the classification) will indicate its pathologic status. Alternatively, the health status of a specimen can be probed by using the synapomorphies as the biomarkers of the disease, that is, through class prediction by assigning the specimen to a clade. Because the cladogram also indicates the direction of change in gene expression among the specimens, it places those specimens with the advanced number of derived gene expression patterns at the terminal end of the cladogram, and places the specimens with the least number of gene expression changes at the lower end of the cladogram, and it may be developed for use in grading, prognosis, targeted treatment, and posttreatment assessment.

Additionally, the phylogenetic classification is a dynamic and seamless tool that will incorporate a novel specimen by placing it in the proximity of its sister groups, depending on the number of synapomorphies it shares with other members of a clade, without any radical alteration to the topology of the cladogram.

\section{Resolving standing questions through parsimony phylogenetics: an example}

Our analysis of uterine fibroids and sarcomas illustrates how a parsimony phylogenetic analysis may confront some of the unresolved issues in bioinformatics and medicine. For example, one of the persistent questions in pathology is the relationship between leiomyoma and leiomyosarcoma (Quade et al., 2004). It has been reported that approximately $1 \%$ of leiomyosarcoma may have arisen in preexisting leiomyoma (Lee et al., 2005). By analyzing data of normal uterus, leiomyoma, and leiomyosarcoma, we demonstrated that the latter two share a number of synapomorphies and form together an inclusive clade (Table 1 and Figs. 1 and 3), and that leiomyosarcoma has an additional number of synapomorphies distinguishing them from leiomyoma (Table 3). Although the leiomyoma specimens, when analyzed alone, without the leiomyosarcoma, appear to have a large number of synapomorphies (Table 2), these synapomorphies are not unique to leiomyoma. Leiomyoma as a group does not form a clade within a comprehensive ingroup that includes the leiomyosarcoma; there is not even one gene expression that is unique to the group itself in this context. Because it shares with the leiomyosarcoma its synapomorphies, leiomyoma may be considered an incipient form of leiomyosarcoma.

\section{Conclusion}

The application of phylogenetic analysis through polarity assessment and parsimony to several gene expression microarray datasets provides the basis for a new paradigm to analyzing and interpreting microarray data (Table 10). It offers an alternative to $F$ and $t$-statistics and fold change methods of generating differentially expressed gene listing and statistical gene linkage, brings out a higher interplatform concordance, resolves interplatform comparability problems, defines biomarkers as synapomorphies, circumscribes disease types as clades defined by synapomorphies, and possibly transforms microarray into diagnostic, prognostic, and posttreatment evaluation tool.

\section{Acknowledgments}

Competing interests: The authors have filed for US patent for their analytical method.

\section{References}

Abu-Asab, M., Chaouchi, M., and Amri, H. (2006). Phyloproteomics: What phylogenetic analysis reveals about serum proteomics. J Proteome Res 5, 2236-2240.

Abu-Asab, M., Chaouchi, M., and Amri, H. (2008). Evolutionary medicine: a meaningful connection between omics, disease, and treatment. Proteomic Clin Appl 2, 122-134.

Adsay, N.V., Merati, K., Andea, A., Sarkar, F., Hruban, R.H., Wilentz, R.E., et al. (2002). The dichotomy in the preinvasive neoplasia to invasive carcinoma sequence in the pancreas: differential expression of MUC1 and MUC2 supports the existence of two separate pathways of carcinogenesis. Mod Pathol 15, 1087-1095.

Albert, V.A. (2005a). Parsimony and phylogenetics in the genomic age. In Parsimony, Phylogeny, and Genomics. V.A. Albert, ed. (Oxford University Press, Oxford, NY).

Albert, V.A. (2005b). Parsimony, phylogeny, and genomics. (Oxford University Press, Oxford, NY).

Alizadeh, A.A., Eisen, M.B., Davis, R.E., Ma, C., Lossos, I.S., Rosenwald, A., et al. (2000). Distinct types of diffuse large Bcell lymphoma identified by gene expression profiling. Nature $403,503-511$.

Allison, D.B., Cui, X., Page, G.P., and Sabripour, M. (2006). Microarray data analysis: from disarray to consolidation and consensus. Nat Rev 7, 55-65.

Beer, D.G., Kardia, S.L., Huang, C.C., Giordano, T.J., Levin, A.M., Misek, D.E., et al. (2002). Gene-expression profiles predict survival of patients with lung adenocarcinoma. Nat Med 8, 816-824.

Bittner, M., Meltzer, P., Chen, Y., Jiang, Y., Seftor, E., Hendrix, M., et al. (2000). Molecular classification of cutaneous malignant melanoma by gene expression profiling. Nature 406, 536-540.

Chung, D.C. (2000). The genetic basis of colorectal cancer: insights into critical pathways of tumorigenesis. Gastroenterology 119, 854-865.

Dong, M., Dong, Q., Zhang, H., Zhou, J., Tian, Y., and Dong, Y. (2007). Expression of Gadd45a and p53 proteins in human pancreatic cancer: potential effects on clinical outcomes. J Surg Oncol 95, 332-336.

Farris, J.S. (1979). The information content of the phylogenetic system. Syst Zool 28, 483-519.

Felsenstein, J. (1989). PHYLIP: phylogeny inference package (version 3.2). Cladistics 5, 164-166.

Felsenstein, J. (2004). Inferring phylogenies. (Sinauer Associates, Sunderland, MA).

Goloboff, P.A., and Pol, D. (2005). Parsimony and Bayesian phylogenetics. In: Parsimony, Phylogeny, and Genomics. V.A. Abert, ed. (Oxford University Press, Oxford, NY).

Golub, T.R., Slonim, D.K., Tamayo, P., Huard, C., Gaasenbeek, M., Mesirov, J.P., et al. (1999). Molecular classification of cancer: class discovery and class prediction by gene expression monitoring. Science 286, 531-537. 
Graybeal, A. (1998). Is it better to add taxa or characters to a difficult phylogenetic problem? Syst Biol 47, 9-17.

Guo, L., Lobenhofer, E.K., Wang, C., Shippy, R., Harris, S.C., Zhang, L., et al. (2006). Rat toxicogenomic study reveals analytical consistency across microarray platforms. Nat Biotechnol 24, 1162-1169.

Guzińska-Ustymowicz, K., Stepień, E., and Kemona, A. (2008). MCM-2, Ki-67 and PCNA protein expressions in pT3G2 colorectal cancer indicated lymph node involvement. Anticancer Res 28, 451-457.

Harrison, A.P., Johnston, C.E., and Orengo, C.A. (2007). Establishing a major cause of discrepancy in the calibration of Affymetrix GeneChips. BMC Bioinformatics 8, 195.

Hayashi, Y., Yamashita, J., and Watanabe, T. (2004). Molecular genetic analysis of deep-seated glioblastomas. Cancer Genet Cytogenet 153, 64-68.

Hippo, Y., Taniguchi, H., Tsutsumi, S., Machida, N., Chong, J. M., Fukayama, M., et al. (2002). Global gene expression analysis of gastric cancer by oligonucleotide microarrays. Cancer Res 62, 233-240.

Hoffman, P.J., Milliken, D.B., Gregg, L.C., Davis, R.R., and Gregg, J.P. (2004). Molecular characterization of uterine fibroids and its implication for underlying mechanisms of pathogenesis. Fertil Steril 82, 639-649.

Huang, K.C., Park, D.C., Ng, S.K., Lee, J.Y., Ni, X., Ng, W.C., et al. (2006). Selenium binding protein 1 in ovarian cancer. Int J Cancer 118, 2433-2440.

Huang, S., and Qu, Y. (2006). The loss in power when the test of differential expression is performed under a wrong scale. J Comput Biol 13, 786-797.

Kolaczkowski, B., and Thornton, J.W. (2004). Performance of maximum parsimony and likelihood phylogenetics when evolution is heterogeneous. Nature 431, 980-984.

Lee, E.J., Kong, G., Lee, S.H., Rho, S.B., Park, C.S., Kim, B.G., et al. (2005). Profiling of differentially expressed genes in human uterine leiomyomas. Int J Gynecol Cancer 15, 146-154.

Lossos, I.S., and Morgensztern, D. (2006). Prognostic biomarkers in diffuse large B-cell lymphoma. J Clin Oncol 24, 995-1007.

Lyons-Weiler, J., Patel, S., Becich, M.J., and Godfrey, T.E. (2004). Tests for finding complex patterns of differential expression in cancers: towards individualized medicine. BMC Bioinformatics 5, 110 .

Meza-Zepeda, L.A , Kresse, S.H., Barragan-Polania, A.H., Bjerkehagen, B., Ohnstad, H.O., Namløs, H.M., et al. (2006). Array comparative genomic hybridization reveals distinct
DNA copy number differences between gastrointestinal stromal tumors and leiomyosarcomas. Cancer Res 66, 8984-8993

Millenaar, F.F., Okyere, J., May, S.T., van Zanten, M., Voesenek, L.A., and Peeters, A.J. (2006). How to decide? Different methods of calculating gene expression from short oligonucleotide array data will give different results. BMC Bioinformatics 7, 137.

Nesse, R.M., and Stearns, S.C. (2008). The great opportunity: evolutionary applications to medicine and public health. Evol Appl 1, 28-48.

Page, R.D. (1996). TreeView: an application to display phylogenetic trees on personal computers. Comput Appl Biosci 12, 357-358.

Pilarsky, C., Wenzig, M., Specht, T., Saeger, H.D., and Grützmann, R. (2004). Identification and validation of commonly overexpressed genes in solid tumors by comparison of microarray data. Neoplasia. 6, 744-750.

Quackenbush, J. (2006). Microarray analysis and tumor classification. New Engl J Med 354, 2463-2472.

Quade, B.J., Wang, T.Y., Sornberger, K., Dal Cin, P., Mutter, G.L., and Morton, C.C. (2004). Molecular pathogenesis of uterine smooth muscle tumors from transcriptional profiling. Genes, Chromosomes and Cancer 40, 97-108.

Siddall, M.E. (1998). Success of parsimony in the four-taxon case: long-branch repulsion by likelihood in the Farris zone. Cladistics 14, 209-220.

Stefankovic, D., and Vigoda, E. (2007). Phylogeny of mixture models: robustness of maximum likelihood and non-identifiable distributions. J Comput Biol 14, 156-189.

Wang, H., He, X., Band, M., Wilson, C., and Liu, L. (2005). A study of inter-lab and inter-platform agreement of DNA microarray data. BMC Genomics 6, 71.

Wiley, E.O., and Siegel-Causey, D. (1991). The Compleat Cladist: A Primer of Phylogenetic Procedures. (Museum of Natural History, Dyche Hall, University of Kansas, Lawrence, KN)

Address correspondence to: Hakima Amri Department of Physiology and Biophysics School of Medicine Georgetown University Washington, DC 20007

E-mail: amrih@georgetwon.edu. 